considerably or completely relieved, and that the psychological benefits outweighed the disadvantages.

As an example of the confusion and ignorance in the public mind, as the basis of so much fear and worry, it is worth noting the results of a recent questionary to the general public reported by Raven (1953)-17\% thought cancer was infectious, $27 \%$ that it was incurable, and $20 \%$ that it was of doubtful curability.

\section{Other Aspects}

In the U.S.A. the average cost per case of cancer discovered has been estimated at anything from 1,000 to 8,000 dollars-clearly prohibitive here. On this score alone, the general practitioner's consulting-room is preferable. Moreover, it is work of a type that enhances the scope of general practice (unlike so many proposals). Conclusions about the value of periodic examinations can be reached only on the basis of large-scale surveys, with adequate follow-up. A suitable agency would appear to be the health centre, of the type recently built, and the financial cost would be relatively low.

\section{Summary}

This paper describes a survey in a general practice, in which clinical medical examination was offered to patients between the ages of 40 and 70 combined with instructional propaganda in early cancer symptoms. Four hundred and twenty-two women and 415 men responded, making acceptance rates of $55 \%$ and $58 \%$. A follow-up examination on the women was also carried out, including a vaginal cytological smear, bringing the total of examinations to about 1,000 .

The findings are reported, with details of malignant and non-malignant lesions discovered.

Some of the arguments for and against such examinations and their possible value in cancer morbidity and mortality are discussed. The psychological and financial aspects are also touched on.

It is concluded that further experimental trials on similar lines would be justified, and are necessary before any appraisal of their long-term value can be made.

Our grateful thanks for the initial grant towards expenses are due to the Sheffield Regional Hospital Management Committee No. 4 (chairman, Sir Ronald Matthews) and its secretary, Captain r. W. Barnard, who gave invaluable help at all stages. Also to the Sheffield Regional Hospital Board for a continuation grant. We would also thank Sir Ernest Finch and Professor Sidney Russ for their interest, encouragement, and help. On the medical side, we are indebted to the kindness of Dr. G. R. Osborn for enabling us to include the vaginal smear examinations; all the material was examined by him. For laboratory assistance we are grateful to Mr. L. R. Reeves, F.I.M.L.T., of the Sheffield National Centre for Radiotherapy, and for secretarial help to Miss B. Wheatley and Mrs. R. Martin.

\section{REFERENCES}

American Cancer Society (1949). Proceedings of First National Cancer Conference, pp. 276-290. New York. Quoted from Cliffton and Rush, (1951).

Boucot, K. R., and Sokoloff, M. J. (1954). Amer. Rev. Tuberc., 69, 164. Cliffton, E. E., and Rush, B. (1951). Surg. Gynec. Obstet., 93, 719.

Discussion (1951). Proc. roy. Soc. Med., 44, 305.

Erickson, C. C. (1953). Quoted from Lancet, 1953, 1, 981

Foulds, L. (1951). Ann. roy. Coll. Surg. Engl., 9, 93

Guiss, L. W. (1952). Cancer (N.Y.), 5, 1035.

Harnett, W. L. (1953). Brit. J. Cancer, 7, 19.

Jones, H. W.. and Cameron, W. R. (1950). J. Amer. med. Ass., 143, 228.

Kreyberg, L. (1953). Brit. J. Cancer, 7, 157.

and Christiansen, T. (1953). Ibid., 7, 37.

McKinnon, N. E. (1952a). Surg. Gynec. Obstet., 94, 173

(1952b). British Medical Journal, 1, 544.

Osborn, G. R. (1953). Applied Cytology. London.

Papanicolaou, G. N and Traut, H. F. (1943). Diagnosis of Uterine Cancer by the Vaginal Smear. New York.

Park, W. W., and Lees, J. C. (1951). Surg. Gynec. Obstet., 93, 129.

Raven, R. W. (1953). Quoted from British Medical Journal, 1953, 1, 502.

Russ, S. (1953). British Medical Journal, 1, 580

Siddall, A. C. (1951). J. Amer. med. Ass., 145, 314

- (1952). Amer. J. Obstet. Gynec., 64, 168.

Swynnerton, B. F., and Truelove, S. C. (1952). British Medical Journal, 1,

Tod, M. (1949). An Inquiry into the Extent to which Cancer Patients in Great Britain Receive Radiotherapy. Sherratt and Hughes, Altrincham. Amer., 21, 1099.
Am. K., and Priestley, J. T. (1941). Surg. Clin. N.

\section{RECENT DEVELOPMENTS IN THE MANAGEMENT OF BRAIN ABSCESS*}

\author{
BY
}

WALPOLE LEWIN, M.S., F.R.C.S.

Assistant Neurological Surgeon, Radcliffe Infirmary, Oxford

During the last ten years there have been considerable advances in the diagnosis and treatment of brain abscess. Of overriding importance has been the introduction of the antibiotics, but superior methods of location, and experience in the methods of treatment available, have all contributed to the improved results. Thus in the Department of Neurological Surgery, Oxford, the mortality has fallen steadily (Table I). The earlier series

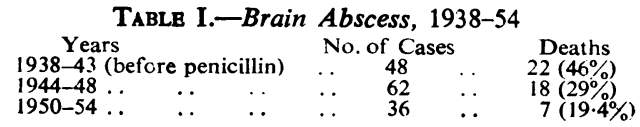

have been described previously (Pennybacker, 1951), and in the present paper the results over the last four years $(1950-4)$ are recorded. During this period there were 36 cases with 7 deaths, a mortality of $19.4 \%$. Concerning these seven deaths it should be noted that three of the patients had been admitted at a late stage, when the abscess had already burst into the ventricle and ventriculitis was established, and one other patient had had multiple abscesses.

In trying to assess these results, the bare figures do not perhaps tell the whole story, since in later years there have been an increasing number of acute cases (including metastatic abscesses, particularly from the chest) reaching neurosurgical clinics for treatment, which may weight the results adversely. The modern trend is perhaps best reflected in the treatment of abscesses resulting from paranasal sinus and otogenic infection : of 20 consecutive patients with abscesses from these sources in the last four years, 19 recovered and 1 died. However, a brain abscess is still a serious matter, and in looking to the future one should not only be seeking for further improvement in diagnosis and treatment but be attempting to reduce the incidence of brain abscess by attention to the sources of infection and also criticizing the quality of the result obtained.

\section{Sources and Prevention}

There is a general impression that brain abscess is less common than used to be the case, and this may well be so. No dramatic change is yet shown, however, in the incidence from neurosurgical clinics, but this may be due to the fact that diagnosis is much better than formerly and more cases are now recognized and sent for treatment.

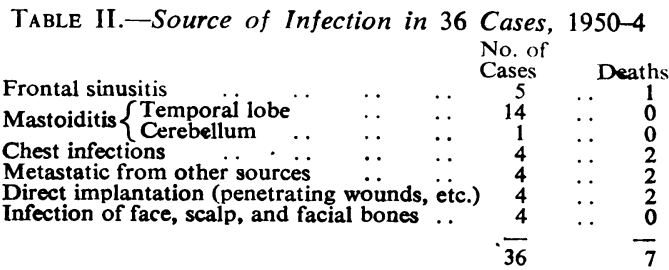

The sources of infection in this series are shown in Table II. The common cause of abscess is paranasal sinus and ear infection, and it would indeed be surprising if the better social conditions of recent years and the greater

* Read in the Section of Neurology at the Annual Meeting of the British Medical Association, Glasgow, 1954. 
attention given to otitis media and the chronic running ear did not lower the incidence of brain abscess. Effective treatment of ear, nose, and throat infections is the most important preventive measure in this field.

Of the 36 cases, 4 resulted from direct wounds of the scalp or skull. The insistence on proper surgical management of all scalp lacerations and compound fractures has yielded rich dividends, and first-intention healing is now so much a routine that there is even a danger of underrating the relatively narrow margin of safety that exists. In a recent patient from Korea, for example, brain abscess followed a small compound depressed fracture of the skull in which definitive surgical treatment was delayed owing to the prior claims of associated severe limb injuries. It was the only case of brain abscess seen among these patients returning to Oxford. $\dagger$ Particular attention is also necessary for fractures of the skull which implicate the paranasal air sinuses and mastoid regions, since they carry an additional risk of intracranial infection (Lewin, 1954).

Before the days of penicillin. metastatic brain abscesses from chest infection such as bronchiectasis and empyema carried a mortality of wellnigh $100 \%$. It is still the most lethal of all the abscesses, but many cases have now been carried through successfully, and indeed the stage has been reached where-for example, after treatment of a brain abscess secondary to bronchiectasis-removal of the affected lung may be undertaken to lessen the chance of a recurrent abscess. A recent series of successful cases so treated has been reported (Pennybacker and Sellors, 1948).

Brain abscess may complicate congenital heart disease even in the absence of bacterial endocarditis, owing to infected venous blood being recirculated through the systemic circulation without first passing through the lungs. Gates et al. (1947) have shown that about $5 \%$ of deaths from congenital anomalies of the heart and great vessels are due to this cause. In the cases recorded, the brain abscess has usually been solitary, so that with the recent developments in cardiac surgery the possibilities of treating the abscess and also the causal lesion are apparent. The risk of precipitating brain abscess by cardiac catheterization in the investigation of congenital anomalies has also been pointed out, and it has been suggested that penicillin and heparin should be given prophylactically.

All these factors, therefore, may combine to lower the incidence of brain abscess in the future. Indeed, in few other instances of serious complications in surgery are the opportunities for preventive measures higher than with brain abscess.

\section{Early Diagnosis}

The clinical picture of the early invasive stage of intracerebral infection with severe headache, vomiting, slowing pulse, and drowsiness passing on to coma is well known, and there is little difficulty in concluding in these circumstances that an intracranial complication has supervened. These gross symptoms and signs due to cerebral oedema and encephalitis still occur, but in many cases nowadays the early use of penicillin so controls this active and very dangerous invasive stage that the first signs of a brain abscess may be attended by only relatively mild changes in the patient's condition. Indeed, the risk is that a diagnosis of cerebral abscess may be excluded on the premise that the patient is not ill enough.

During a sinus or mastoid infection, the very fact that the patient is not as well as he ought to be should be enough to raise the suspicion of an intracranial complication, and this probability is increased if headache is present. It is at this stage that a careful neurological examination may yield unequivocal evidence. Obvious papilloedema, a hemiparesis, dysphasia, or frank ataxia may be present, but again in other patients such gross signs are absent. Frontal lobe abscesses, for example, may declare themselves by only a

†The patients from Korea were admitted to the Military Hospital (Head Injuries), Wheatley, Oxon, and their management will be described elsewhere. mild reflex change down one side of the body, or possibly a little facial weakness or an isolated fit, or nocturnal incontinence in a patient otherwise alert. In the temporal lobe, an abscess will very commonly produce a visual-field defect in the opposite upper homonymous field, which may be missed unless confrontation tests and careful perimetry are done as a routine; and if it is in the dominant hemisphere a dysphasia may be present, but may be mild and detected only if formal examination such as the meaning of objects, etc., is carried out. A cerebellar abscess usually gives rise to nystagmus. These relatively mild signs, when looked for, are present much more often than is thought to be the case.

Lumbar Puncture.--A lumbar puncture may be invaluable, since the fluid is rarely normal in the presence of an acute or subacute abscess. The protein content is usually raised to $100 \mathrm{mg}$. per $100 \mathrm{ml}$. or more (normal $40 \mathrm{mg}$.) and there is often an increase of white cells in the C.S.F. from 10 to 200 per c.mm. Nevertheless this test should be used with caution in the presence of marked papilloedema, a slow pulse, or rapidly increasing drowsiness, since there is a risk of tentorial impaction, and in these circumstances it is wise to have facilities for dealing with the abscess at hand.

Electroencephalography and angiography are two other ancillary methods of diagnosis that have been developed in recent years and are proving helpful in the more accurate location of an abscess.

Electroencephalography.-This test may help in locating an abscess and also in differential diagnosis from such conditions as thrombophlebitis. In addition, serial recordings during the management of an abscess may give a clue should a further abscess be developing elsewhere, particularly in cases of metastatic origin. The focal disturbance seen is that common to any rapidly expanding intracranial lesion, and consists essentially of a high-voltage delta activity in the vicinity of the lesion, together with a local phase reversal. Tutton and Whittaker (1953, personal communication) have commented favourably on the use of the E.E.G. in the management of brain abscess. It has the merit of being easy to perform and not disturbing to the patient.

Angiography.-Another recent development in the diagnosis of brain abscess has been the use of angiography. For supratentorial lesions, particularly of the frontal and temporal lobes, which are the common sites for abscess, it may be invaluable and will locate the lesion accurately without the considerable upset that may attend the use of ventriculography in such cases. It affords, of course, no evidence of the true nature of the lesion, and in abscess cases the appearance is one of vessel displacement by an avascular space-occupying lesion.

Ventriculography is still used for the diagnosis of the difficult deeply seated hemisphere and infratentorial abscess, but the tendency nowadays is to employ air studies less in the acute stage, although, as referred to below, to rely on them, if anything, more in the later stages as a test of cure.

\section{Treatment}

Now that the antibiotics, usually penicillin, do so much to control the invasive stage of encephalitis and cerebral oedema, it is possible to simplify the surgery of the acute abscess, and most surgeons are agreed to-day that the first treatment of choice, and one which is applicable to the majority of abscesses, is simple needle aspiration of the abscess through a suitably placed burr-hole, repeated as required, with local injection into the abscess cavity of the appropriate antibiotic-in most cases penicillin (Tutton, 1953). Taylor (1950) favours in addition the routine use of intraventricular penicillin during this stage, and makes the point that it lessens the risks of dissemination of the infection into the cerebrospinal fluid spaces of the brain around the abscess, one of the main complications in the management of brain abscess. 
Part of the success of aspiration is due to the accurate location of the abscess, and the advantages of the recent developments already referred to are apparent. The operation is conducted through a clean incision, and we have long since abandoned attempts to drain an abscess along the entry track, as, for example, through a mastoid wound, which may lead to cerebral fungus. Similarly, open drainage is rarely effective after the first escape of pus, and the insertion of tubes and subsequent manipulations may cause further brain destruction and risk of rupture of the abscess ; in addition it may leave undrained a loculus of the abscess.

In a few cases aspiration with local and systemic antibiotics is inadequate to control the raised intracranial pressure during the acute stage and a decompression may also be required. In addition there are other abscesses, especially those resulting from penetrating wounds or metastatic from the thorax, in which a honeycomb of small abscesses or a complicated abscess cavity may result, for which aspiration or tube drainage proves inadequate, and in this instance excision of the phlegmon in the acute stage may be indicated. Indeed, Le Beau (1946) has advocated its wider adoption, but we would restrict this operation to those few cases in which there is no other effective alternative, since it must mean sacrifice of recoverable cerebral tissue and an increased risk of resultant disability.

Thus, although one can see a general plan of treatment emerging, there is no one operation which is applicable to all acute abscesses. To improve the results of this stage therefore requires that the surgeon should have all these methods at his disposal and consider each case on its merits. Even so, the risks of rupture of the abscess into the ventricle, as shown from the deaths in this series, and the problem of multiple abscesses remain major problems. Thus in one of two recent fatal cases the patient had a left metastatic parietal abscess treated by aspiration, supplemented later by a decompression when it was clear that more was required, but at necropsy a second abscess with gross surrounding oedema was found further forward, deep in the post-frontal region, and a third on the opposite side. In the other case (not in this series) small quantities of pus were aspirated from both frontal lobes of a patient who developed signs of cerebral infection with no obvious primary focus other than an upper respiratory infection which had resolved. At necropsy it was possible to count at least 130 small abscesses scattered through the hemispheres. These two examples may be uncommon, but they serve as a salutary reminder that the treatment of the acute abscess is unlikely to become a matter of routine.

The management of the later stages is still debatable. Most abscesses can be treated by aspiration alone in the acute stage, and some surgeons believe that if further aspirations yield no pus, and provided the patient is well, no more need be done, although the majority agree that, as a final test of cure, air studies should be carried out in the quiescent stage to ensure that no loculus remains and that the lumbar C.S.F. protein and cell content have returned to normal. This policy is attractive in its simplicity and treats the abscess with the least possible damage to the surrounding brain, and, presumably, therefore lessens the risks of ultimate scarring.

There are difficulties, however. If this method alone is to be used then the tests of cure have to be rigid; most surgeons have had the experience of the well patient with only a doubtful abnormality in the ventricular air studies ahd yet shown subsequently to have a large abscess. Indeed, the gross displacements seen in ventriculograms and angiograms during the acute stage are due as much to the oedema around an abscess as to the amount of pus (Schurr, 1951); and subsequently a sizable collection may remain in the frontal pole or the inferior part of the temporal lobe or the cerebellum, with the production of only minimal radiographic changes. Moreover, a negative aspiration does not exclude the presence of pus : in some instances the pus becomes so thick as not to flow through the needle; or a loculus, which occurs in over $40 \%$ of abscesses, may be present and remain untapped; or the abscess may be missed altogether. Further, we have seen several examples where sudden and dangerous enlargement of an abscess has occurred in a patient previously doing well.

For these reasons, therefore, other surgeons have injected radio-opaque substances into the abscess cavity at the time of aspiration so that its subsequent fate may be followed. Detection of an unsuspected loculus or enlargement of the abscess may then allow a timely aspiration before dangerous complications supervene. For many years now this has been our policy, and we have used thorotrast. Oliver and Leese (1949), to overcome the objection of introducing a substance which is retained in the abscess wall, have suggested the use of diodone, which is absorbed; but this method does not confer the real advantage of using a radio-opaque substance-namely, to watch the progress of the abscess and be able to anticipate developments.

Thorotrast is of course a foreign material, and Taylor (1950) thinks that it may promote chronicity. It has also been suggested that it may increase the fibrous reaction around the abscess. The substance is also radioactive. We have not seen any obvious differences in the brain around an abscess at the time of excision whether it has previously been injected with thorotrast or not, nor, in the amounts used, have we so far seen any adverse effects from possible radioactivity. The substance has now been used for this purpose for about 14 years, so that presumably the risk is a small one. It is possible that in the future early diagnosis and effective antibiotics will make loculation of abscesses and other complications of management less common and that in some cases at least it will be possible to dispense with this additional guide. If so, it would be welcome, but at present one would feel that, as further improvement in results is likely to come from attention to individual details, the surgeon needs all the help he can get in a condition so dangerous and treacherous as brain abscess.

\section{Excision}

Apart from those particular abscesses which may require excision in the acute stage, there are others in which pus continues to reaccumulate despite repeated aspiration. In some instances this is related to the sensitivity of the causal organism to antibiotics, and careful bacteriology is, of course, an essential part of the aspiration-antibiotic regime. Nevertheless some of the most troublesome abscesses to deal with are those in which all cultures are persistently sterile, yet the behaviour of the abscess is evidence that the infection is anything but quiescent, and for these cases excision may be required.

How safe is the patient from recurrent infection when treated by aspiration alone and when it is realized that $40 \%$ of abscesses are multilocular? In a recent review of 295 abscess cases from Edinburgh, Manchester, and Oxford, Jooma et al. (1951) found that, of 88 survivors treated by aspiration or drainage alone, 7 developed recurrent infection $(8 \%)$ at intervals of from 3 months to 13 years later, and they all died; among 96 patients who survived excision there were no recurrences. These figures should not be overemphasized, since much of the material was in the pre-penicillin era, and one would anticipate that, with recent developments and the more rigid standards of cure in the future, this incidence would fall. Nevertheless, it is clear that only by excision of the whole area can one be certain of ultimate cure. Provided, therefore, that it can be done without increasing the patient's disability, or materially increasing the mortality, excision requires consideration. We have followed this policy in the past. An added hope was that by removal of the abscess scar the incidence of epilepsy later on would be significantly lowered As referred to below, this has only been partly realized. It is certainly true that, with the more successful management of the acute abscess by aspiration and antibiotics, fewer abscesses will need excision in the future, but there are still cogent reasons for advising excision of frontal, 
temporal, and cerebellar abscesses when there is the slightest doubt that the infection has not resolved. In the last four years we have excised 18 abscesses $(62 \%$ of the survivors from the acute stage) with no deaths.

\section{The Primary Focus}

The primary focus of infection needs to be considered in every case, although in some it has resolved before the brain abscess is evident and in others it responds to the antibiotics given during treatment. When surgery is required it is generally agreed that the brain abscess should first be brought under control. In cases of sinus infection or mastoiditis which require drainage this can then be carried out shortly afterwards with greater safety or, in other cases, deferred into the convalescent stage. More elective treatment, such as lobectomy for bronchiectasis, is usually carried out when treatment for the brain abscess is finished.

\section{Sequelae}

The late effects of cerebral abscess are three : recurrent infection, which has already been discussed, permanent neurological disability, and epilepsy. It is not proposed to discuss the permanent effects in detail except to say that grave disabilities are happily uncommon. Of the 29 survivors of the last four years, only two are permanently disabled. In one disablement was due to a decerebrate state which resulted from brain-stem compression, the patient being admitted in coma with fixed dilated pupils; in the other patient a progressive hydrocephalus developed secondary to meningitis. which accompanied the abscess. Indeed, it is remarkable how ill and handicapped these patients can be in the acute stage and yet ultimately make good functional recoveries.

Epilepsy, however, remains the major problem in patients with abscesses of the cerebral hemispheres; it develops in over $45 \%$ of the survivors. Indeed, with recovery from brain abscess becoming more the rule, this complication is likely to be more apparent to the surgeon in the future. Nevertheless, one should hasten to add that in most cases the attacks are infrequent, and in only a few are they of such an order as to render the patient unemployable. Its incidence is highest with multiple abscesses and with frontal abscesses, being well over $50 \%$, and is least with temporal abscesses, $34 \%$. Fortunately with the wide range of anticonvulsant drugs now available, the majority of patients are able to lead a normal life with perhaps an occasional attack, the tendency being for the attacks to diminish as time goes on. Nevertheless our aim should be to reduce its incidence if possible, and in this connexion there are at least two possibilities.

If one believes that it is the scar left after the abscess has healed, rather than the very fact of having had an acute infection of the brain, that is responsible for the epilepsy, then the smaller the scar the better, and it may be that, in the future, early diagnosis and antibiotics will lower its incidence. Again, if it is the scar that is responsible, would not excision lower the incidence of subsequent fits? Jooma et al. (1951) showed that the incidence of epilepsy was significantly lower after excision of a frontal abscess $(44 \%)$ than after treatment by aspiration or drainage alone $(72 \%)$. The fits after a frontal abscess are particularly important, since they are the more dangerous as they may occur without warning, and in this site also status epilepticus is likely. Indeed, in the review by Jooma et al. (1951) there were five fatal cases in the late results from status epilepticus, and all were frontal abscesses. On these results, therefore, there would appear to be a good case for excision of a frontal abscess on the grounds of epilepsy alone.

Temporal lobe abscesses also show a lower incidence of epilepsy after excision (27\%) compared with aspiration alone $(40 \%)$, but the practical results are not so striking, since disabling epilepsy from this site is uncommon. The explanation for this improvement with excision may lie in the fact that in excising a frontal abscess one is usually able to take a more generous excision into the surrounding white matter and the track is usually excised as well as the abscess cavity, so that in effect a scar excision, as in operating for traumatic epilepsy, is not infrequently performed. To some extent this may also be true of some temporal lobe abscesses, particularly in the non-dominant hemisphere. It may therefore be that in suitable cases electrocorticography and removal, where practicable, of active foci in addition to excision of the abscess should receive further consideration. Certainly surgery directed to the epilepsy as in posttraumatic cases should yield results, either in the phase of active treatment or later on if epilepsy is declared and is proving a major handicap, which are at least comparable.

\section{Summary and Conclusions}

The advent of antibiotics, the wider recognition of the early signs of intracranial infection, and the use of ancillary methods of investigation such as angiography and electroencephalography have all contributed to the improved results in the treatment of brain abscess. In the series of 36 cases reported here over the last four years there were seven deaths, a mortality of $19.4 \%$. Prevention, however, is better than cure, and the several ways by which this may be partly achieved are outlined.

Intermittent aspiration of the abscess combined with local and systemic antibiotics, usually penicillin, is recommended as the treatment of choice in the acute stage, but formal decompression may be required in certain cases. The indications for excision of the abscess both in the acute and in the later stages are also discussed.

The various ancillary methods of investigation are valuable both in differential diagnosis and in the management of a brain abscess, but it is wise to remember that in some instances the results they yield are at the best equivocal and that in dealing with intracranial infection a sound clinical appreciation of the case remains the first essential.

There are at least two major problems which call for further study. One is the management of the metastatic abscess, especially that from the chest. Frequently multiple, often multilocular, neither easy to diagnose or locate nor necessarily occurring in sites readily accessible to surgery, it remains a challenge to the surgeon. The other problem is the high incidence of epilepsy $(45 \%)$ among the survivors from brain abscess. It may be that its incidence in the future will fall with improved methods of treatment in the acute stage, but the possibilities of surgical excision with the help of electrocorticography, particularly in the frontal region, merit further attention.

The recent advances in the management of brain abscess should be a stimulus to lower the mortality further and to improve the quality of the result.

My thanks are due to Mr. Joe Pennybacker for his helpful criticism in the preparation of this paper.

\section{REFERENCES}

Gates, E. M.. Rogers, H. M., and Edwards, J. E. (1947). Proc. Mayo (lua 22. 401 .

Jooma, O. V., Pennybacker, J. B., and Tutton, G. K. (1951). J. Neurot. Neurosurg. Psychiat., 14, 308.

Le Beau. J. (1946). J. Neurosurg., 3, 359

Lewin, W. (1954). Brit. J. Surg., 42, 1.

Oliver. L. C.. and Leese. W. L. B. (1949). Lancet. 2. 828.

Pennybacker. J. B. (1951). In Modern Trends in Neurology, edited by A Feiling. Butterworth, London.

- and Sillors, T. Holmes (1948). Lancet, 2, 90.

Schurr, P. H. (1951). Brit. J. Surg., 39, 156.

Taylor, J. (1950). Proc. roy. Soc. Med., 43, 129.

Tutton, G. K. (1953). Ann. roy. Col. Surg. Engl., 13, 281. 